\title{
Universal features of the off-equilibrium fragmentation with the Gaussian dissipation
}

\author{
Robert Botet $^{\dagger}$ and Marek Płoszajczak ${ }^{\ddagger}$ \\ † Laboratoire de Physique des Solides, Bâtiment 510, Université Paris-Sud \\ Centre d'Orsay, F-91405 Orsay, France \\ and \\ $\ddagger$ Grand Accélérateur National d'Ions Lourds (GANIL), \\ CEA/DSM - CNRS/IN2P3, BP 5027, F-14076 Caen Cedex 05, France
}

(October 3, 2018)

\begin{abstract}
We investigate universal features of the off-equilibrium sequential and conservative fragmentation processes with the dissipative effects which are simulated by the Gaussian random inactivation process. The relation between the fragment multiplicity scaling law and the fragment size distribution is studied and a dependence of scaling exponents on the parameters of fragmentation and inactivation rate functions is established.
\end{abstract}

PACS numbers: $64.60 . \mathrm{Ak}, 05.40 .+\mathrm{j}, 82.20 . \mathrm{Mj}$

Typeset using REVTEX 
Fragmentation is the universal process which can be found at all scales in the nature. The most general sequential binary and conservative fragmentation processes with scaleinvariant fragmentation and inactivation rate functions, have been previously studied in much details [1-3]. The phase diagramme of these off-equilibrium processes has been established and the universal aspects of both the fragment size distribution and the total number of fragments distribution (i.e., the multiplicity distribution) have been determined [3,4] . In this fragmentation-inactivation binary (FIB) model [1, 2], one deals with fragments characterized by some conserved scalar quantity that is called the fragment mass . The anscestor fragment of mass $N$ is fragmenting via an ordered and irreversible sequence of steps. The first step is either a binary fragmentation, $(N) \rightarrow(j)+(N-j)$, or an inactivation $(N) \rightarrow(N)^{*}$. Once inactive, the cluster cannot be reactivated anymore. The fragmentation leads to two fragments, with the mass partition probability $\sim F_{j, N-j}$. In the following steps, the process continues independently for each active descendant fragment until either the low mass cutoff for further indivisible particles (monomers) is reached or all fragments are inactive. For any event, the fragmentation and inactivation occur with the probabilities per unit of time $\sim F_{j, k-j}$ and $\sim I_{k}$ respectively. The fragmenting system and its evolution is completely specified by that rate functions and the initial state. It is also useful to consider the fragmentation probability $p_{F}$ without specifying masses of descendants $: p_{F}(k)=\sum_{i=1}^{k-1} F_{i, k-i}\left(I_{k}+\sum_{i=1}^{k-1} F_{i, k-i}\right)^{-1}$. If the instability of smaller fragments is smaller than instability of larger fragments, $p_{F}(k)$ is an increasing function of fragment mass and the total mass is converted into finite size fragments. This is the shattered phase. The fragment mass independence of $p_{F}(k)$ at any stage of the process until the cutoff-scale for monomers characterizes the critical transition region. The multiplicity anomalous dimension $: \gamma=d(\ln <m>) / d(\ln N)$, is the order parameter in the FIB model. It equals 1 in the shattering phase and takes the intermediate value between 0 and 1 in the critical transition region.

For most fragmenting systems, the off-equilibrium relaxation process ceases due to a dissipation. The dissipation is not always scale-invariant as considered in Ref. 1 but, on 
the contrary, it is often characterized by a definite and usually small length scale. It is then an open question to which extent the fragmentation processes which on one side are driven by the homogeneous scale-invariant fragmentation rate function and on other side are inactivated at a certain fixed scale by the random inactivation process, may develop scale-invariant and universal features in both the fragment mass distribution $n(k)$ and the fragment multiplicity distribution $P(m)$. This question is important in view of the widespread occurence of scale-invariant fragment mass distributions $n(k) \sim k^{-\tau}$ and the lack of convincing arguments for using homogeneous dissipation functions in many processes including parton cascading in the perturbative quantum chromodynamics (PQCD) [5] or the fragmentation of highly excited atomic nuclei, atomic clusters or polymers. In this work, we address this fundamental question using the FIB process with the homogeneous fragmentation rate function : $F_{j, k-j}=[j(k-j)]^{\alpha}$, and with the dissipation at small scales which is modelled by the Gaussian inactivation rate function :

$$
I_{k}=c \exp \left[-\frac{1}{2 \sigma^{2}}\left(\frac{k-1}{N}\right)^{2}\right]
$$

An asymptotic $(t \rightarrow \infty)$ fragment mass distribution in the critical transition region of FIB model with scale-invariant dissipation phenomena [1],2], is a power law with an exponent $\tau \leq 2$. In the shattering phase, the fragment mass distribution is also power law but with an exponent $\tau>2$. Another characteristic observable is the fragment multiplicity distribution : $P(m)=\sum_{k} P_{k}(m)$, where $P_{k}(m)$ is the probability distribution of the number of fragments of mass $k$. This quantity has been intensely studied in the strong interaction physics [6]. Of particular importance is a possibility of asymptotic scaling of multiplicity probability distributions :

$$
<m>^{\delta} P(m)=\Phi\left(z_{(\delta)}\right), \quad z_{(\delta)} \equiv \frac{m-<m>}{<m>^{\delta}}
$$

where the asymptotic behaviour is defined as $<m>\rightarrow \infty, m \rightarrow \infty$ for a fixed $(m /<m>)$ - ratio. $<m>$ is the multiplicity of fragments averaged over an ensemble of events. The scaling law (2) means that for example data for differing energies (hence 
differing $\left\langle m>\right.$ ) should fall on the same curve when $<m>^{\delta} P(m)$ is plotted against the scaled variable $z_{(\delta)} \equiv(m-<m>) /<m>^{\delta}$. Some time ago Koba, Nielsen and Olesen (KNO) suggested an asymptotic scaling (22) with $\delta=1$ in the strong interaction physics [7]. The same scaling has been found also in the critical transition region of scale-invariant FIB process for $p_{F}>1 / 2$ and $\alpha \geq-1$ [3]. Recently, Botet, Płoszajczak and Latora (BPL) reported another scaling limit in (2) with $\delta=1 / 2$, which holds in the percolation and in the shattering phase of scale-invariant FIB process 诸 $. \delta=1 / 2$ and 1 are the two limiting values since $\delta>1$ or $\delta<1 / 2$ are incompatible with the scaling hypothesis (2) .

The study presented in this Letter correspond to the domain $\alpha \geq-1$ of fragmentation rate functions $F_{j, k-j}$. Many known homogeneous fragmentation kernels correpond to this domain. These include the singular kernel $\alpha=-1$ in the PQCD gluodynamics [ , $\alpha=-2 / 3$ for the spinodal volume instabilities in three dimensions [2], $\alpha=+1$ in the scalar $\lambda \phi_{6}^{3}$ field theory in six dimensions [9], and many others [2] . For $\alpha<-1$, the fragmentation process is dominated by the splitting $(k) \rightarrow(k-1)+(1)$ at each step in the cascade, and leads to the finite limiting value of $\langle m\rangle$ independently of the initial size $N$ [3] . In this evaporation phase, the scaling solution (2) does not hold and the multiplicity anomalous dimension is equal zero when $N \rightarrow \infty$. This phase is not relevant for the problem we want to address in this Letter.

Without restricting the generality of our discussion, we will present below results for fragmentation kernels with : $\alpha=-1$ and $\alpha=+1$. The upper part of Fig. 1 shows multiplicity distributions for $\alpha=-1$ in the scaling variables (2) for $\delta=1$ (the upper left part), and fragment mass distributions for the same parameters (the upper right part). The cascade equations of Gaussian FIB model have been solved by Monte-Carlo simulations [1:2] for different initial system sizes $(N=1024,4096)$ and for the following exemplaric parameters : $c=1$ and $\sigma=0.1,1$ of inactivation rate function $I_{k} \equiv I_{k}(c, \sigma)$. We have made exhaustive analysis of $P(m)$ for a broad range of $c, \sigma$ parameters, finding in all cases the KNO scaling $(\delta=1)$. We have found the KNO scaling uniquely for $\alpha=-1$. The shape of KNO scaling function $\Phi\left(z_{(1)}\right)$ depends on the precise value of both $c$ and $\sigma$. 
In the lower left part of Fig. 1, we show typical multiplicity distributions for $\alpha=$ +1 which are plotted for different system sizes in the BPL scaling variables $(\delta=1 / 2)$. The corresponding fragment mass distributions are shown in the lower right part of Fig. 1. Again, the precise form of BPL scaling function $\Phi\left(z_{(1 / 2)}\right)$ depends on the chosen set of parameters $c$ and $\sigma$. In contrast to these results of Gaussian FIB model, fragmentation process in the scale-invariant FIB model for any value of exponent $\alpha$ may be found either in the critical transition region or in the shattering phase depending on the homogeneity index $\beta$ of the inactivation rate function $I_{k}=I_{1} k^{\beta}$ [1,2]. This means that e.g. both for $\alpha=-1$ and +1 , one may see either the KNO scaling or the BPL scaling of multiplicity distributions depending on the precise value of the homogeneity index of the inactivation term.

Concerning the fragment mass distributions, Fig. 1 shows the distributions for $\alpha=$ $-1,+1$ and different parameters of Gaussian inactivation rate function $I_{k}(c, \sigma)$. For $\sigma$ larger than $\sim 0.5$, one finds the power law distribution of fragment masses for any value of parameter $c$. In the studied case : $\sigma=1, c=1$, the exponent $\tau$ equals 1.8 and 2.8 for $\alpha=-1$ and $\alpha=+1$ respectively. For a given $\alpha$, the value of exponent $\tau$ is remarkably independent of $\sigma$ but depends strongly on the value of parameter $c$ in $I_{k}(c, \sigma)$. For a smaller value of $\sigma(\sigma=0.1$ is shown in Fig. 1), the fragment mass distribution decreases exponentially and the shape of scaling function resembles the Gaussian distribution. The form of this exponential distribution depends both on $c$ and $\sigma$ parameters.

As a generic case for $\alpha=-1$, we have found the scale-invariant region of power law fragment mass distributions with $\tau \leq 2$ for $\sigma$ above $\sim 0.5$, and the exponential region of mass distributions for $\sigma$ less than $\sim 0.5$. The power law region is completely analogous to the critical transition region of scale-invariant FIB model for $\alpha>-1$ and $p_{F}>1 / 2$ [1-3], because the multiplicity anomalous dimension in both models is :

$$
\gamma=\tau-1 \quad(0 \leq \gamma \leq 1)
$$

We have verified validity of this relation in Gaussian FIB model for a broad range of $c, \sigma$ val- 
ues. In the exponential region,$\gamma$ is always equal 1 independently of the value of parameter $c$, i.e. this region is in the shattering phase. One should remind that shattering in the scaleinvariant FIB model is related exclusively with the BPL scaling, whereas in the Gaussian FIB model for $\alpha=-1$ the KNO scaling holds.

The fragment size distributions for $\alpha=+1$ and different values of $\sigma$ behave similarly as for the $\alpha=-1$ case, except that now for $\sigma$ above $\sim 0.5$ the power law exponent is $\tau>2$. For all $\sigma$, i.e. in both exponential and power law regions of mass distribution, the multiplicity anomalous dimension is $\gamma=1$ and the BPL scaling holds. This generic situation is completely analogous to the multiplicity behaviour found in the shattering phase of scale-invariant FIB model [1],2] .

Whenever the fragment size distribution is a power law, the KNO scaling of multiplicity distributions is associated with $\tau \leq 2$ and the BPL scaling of multiplicity distributions with $\tau>2$ in both scale-invariant and scale-dependent regimes of dissipation. This clearly indicates a direct relation between the multiplicity scaling law and the fragment mass distribution scaling regimes in the FIB model. In view of the generality of FIB process, it would be very interesting to test this relation experimentally. A novel aspect of the Gaussian FIB model is associated with properties of multiplicity scaling in the new region of exponential fragment mass distributions. In this region, BPL scaling holds for $\alpha=+1$ whereas KNO scaling is seen for $\alpha=-1$.

In Fig. 2 we plot for different values of the parameter $c$ the normalized cumulant factorial moment of order two [10]: $\gamma_{2}=\left(<m(m-1)>-<m>^{2}\right) /<m>^{2}$, vs the width $\sigma$ of inactivation rate function $I_{k}(c, \sigma)$. The exponent of homogeneous fragmentation kernel is : $\alpha=-1$. For this choice of $\alpha$, the KNO scaling holds and $\gamma_{2}$ becomes the second moment of scaling function $\Phi\left(z_{(1)}\right)$ which is independent of initial mass $N$ [6, 3] . For each point $(c$, $\sigma$ ), cascade equations of the FIB model have been solved exactly by recurrent formula [3] up to initial system size $N=2^{18}$. As can be seen in Fig. 2, the multiplicity fluctuations as measured by $\gamma_{2}$ are extremely small in the exponential region for $\sigma$ less than $\sim 0.5$. The change of $\gamma_{2}$ when passing from the power law to exponential region is continuous but the 
largest variations of $\gamma_{2}(\sigma)$ appear at $\sigma \sim 0.5$. For large values of $\sigma$, the cumulant factorial moment approaches a limiting value which depends on the value of parameter $c$.

The experimental informations about $\gamma_{2}$ are not numerous and concern mainly charged particle multiplicities at relativistic and ultrarelativistic energies. The DELPHI Collaboration reported the data on hadron production in $e^{+} e^{-}$annihilations for the center of mass (c.m.) energy of $\sqrt{s}=91 \mathrm{GeV}$ finding $\gamma_{2}=0.04$ [11]. In hadron-hadron collisions $\pi^{+}-p$, $K^{+}-p, p-p, p-\bar{p}$ for c.m. energies ranging up to $1000 \mathrm{GeV}$ [6, 12], values of $\gamma_{2}$ increase from about 0.05 to 0.3 as energies increase to collider values. Distribution of galaxy counts in the regions of sky covered by the Zwicky catalogue [13] yields $\gamma_{2} \simeq 0.3$ [14]. Independently of the question whether the KNO scaling holds in all those different physical systems , the measured values of $\gamma_{2}$ clearly exclude the exponential region of Gaussian FIB process. Much more information could be extracted if in addition to the moments of the multiplicity distribution also the mass distribution would be available. In high energy lepton and/or hadron collisions for example, this would require measuring the hadron mass distribution.

In conclusion, we have demonstrated that the off-equilibrium binary fragmentation with scale-invariant fragmentation kernel and the scale-dependent inactivation simulating the dissipation at small scales, yields the fragment mass and fragment multiplicity distributions which are scale-invariant for a broad range of parameters. This is an important finding because most of fragmentation processes in nature which have these scale-invariant features are probably not associated with the dissipative processes acting at all scales. The scaledependent fragmentation processes may also develop strong scale-invariant fluctuations (the KNO scaling) though the region of their appearance is restricted to the particular value of exponent : $\alpha=-1$, of the homogeneous fragmentation function. The region at $\alpha=$ -1 and $\sigma$ above $\sim 0.5$ is the critical transition region of Gaussian FIB process. For other values of $\alpha$, the fragment multiplicity distributions obey the BPL scaling, i.e. the small amplitude limit of scaling multiplicity fluctuations. Another transition zone of the Gaussian FIB model is defined by the width $\sigma$ of inactivation rate function. At $\sigma \simeq 0.5$, the fragment size distribution changes from exponential (for $\sigma<0.5$ ) into power law (for 
$\sigma>0.5)$. The form of scaling function $\Phi\left(z_{\delta}\right)$, together with the form of fragment mass distribution $n(k)$ impose strong constraints on the choice of basic functions of FIB kinetic equations : the fragmentation and inactivation functions. This has been demonstrated on the example of hadron production data in the $e^{+} e^{-}$annihilation [15] . Results of this Letter show that the closing of gap between experimental observables related to the fragment mass distribution and/or the fragment multiplicity distribution and the basic ingredients of the kinetic theory, i.e. the rates of activation $F_{j, k-j}$ and inactivation $I_{k}$, can be achieved for many physical systems in the nature. 


\section{REFERENCES}

[1] R. Botet and M. Ploszajczak, Phys. Rev. Lett. 69, 3696 (1992).

[2] R. Botet and M. Ploszajczak, Int. J. of Mod. Phys. E3, 1033 (1994).

[3] R. Botet and M. Ploszajczak, Phys. Rev. E54, 3320 (1996).

[4] R. Botet, M. Ploszajczak and V. Latora, Phys. Rev. Lett. 78, 4593 (1997).

[5] R. Botet and M. Ploszajczak, Proc. 7th Int. Workshop on Multiparticle Production Correlation and Fluctuations, Nijmegen, The Netherlands, June 30 - July 6, 1996, R.C. Hwa, E.W. Kittel, W.J. Metzger and D.J. Schotanus eds. (World Scientific, 1996).

[6] P. Carruthers and C.C. Shih, Int. J. Mod. Phys. A2, 1447 (1987).

[7] Z. Koba, H.B. Nielsen and P. Olesen, Nucl. Phys. B40, 317 (1972).

[8] Yu.L. Dokshitzer, V.A. Khoze, A.H. Mueller and S.I. Troyan, Basics of Perturbative QCD, ed. Tran Thanh Van (Editions Frontieres, Gif-sur-Yvette, 1991).

[9] I.M. Dremin, Physics - Uspekhi 37, 715 (1994).

[10] The factorial cumulant moments are integrals of corresponding cumulants which measure physical density correlations free from trivial background of lower order density correlations [6] .

[11] P. Abreu et al. (DELPHI Collaboration), Z. Phys. C 50, 185 (1991).

[12] G. Giacomelli, Invited paper at the 1989 Int. Workshop on Multiparticle Dynamics, In honour of Leon Van Hove, La Thuile, Valle d'Aosta, Italy, Preprint CERN-EP/89-179

[13] F. Zwicky, E. Herzog, P. Wild, M. Karpowicz and C.T. Kowal, Catalog of galaxies and clusters of galaxies, Vols. 1-6 (California Institute of Technology, Pasadena, 1961-1968).

[14] P. Carruthers and Minh Duong-Van, Phys. Lett. 131B, 116 (1983).

[15] R. Botet and M. Ploszajczak, Z. Phys. C76, 257 (1997). 


\section{Figure captions}

\section{Fig. 1}

Multiplicity probability distributions in the scaling variables (see eq. (2)), and the fragment mass distribution for two homogeneous fragmentation kernels and two Gaussian inactivation rate functions. Each set of data corresponds to $10^{6}$ independent events of Monte-Carlo simulations.

(i) Upper left part : the fragmentation kernel with $\alpha=-1$ and the inactivation rate function (11) for $c=1$ and two typical values of $\sigma$. Two sets of data are plotted for two different total mass : $N=1024$ (crosses) and $N=4096$ (circles). These data are plotted in the KNO form, i.e. : $\delta=1$ (see eq. (国)).

(ii) Upper right part : the fragment mass distributions in a double-logarithmic scale are shown for the same parameters $\alpha, c, \sigma$ as in (i). The total mass is $N=4096$. Big stars represent results obtained for the same value of $\alpha, c$ parameters and for a much larger value of $\sigma(\sigma=10)$, to show the independence of the scaling part of the fragment mass distribution with the value of $\sigma$. The line in between points is shown to guide the eyes.

(iii) Lower left part : the same as in (i) but for the fragmentation kernel with $\alpha=+1$. These data are plotted in the BPL form, i.e. : $\delta=1 / 2$ (see eq. (2)).

(iv) Lower right part : the fragment mass distributions for $\alpha=+1$. Parameters $c, \sigma, N$ as in (ii).

\section{Fig. 2}

The cumulant factorial moment $\gamma_{2}$ of the fragment multiplicity distribution is plotted vs the width parameter $\sigma$ of the Gaussian inactivation function (1) with $c=0.5,1,5$. The homogeneous fragmentation kernel is taken with $\alpha=-1$. Each point corresponds to system of size $N=2^{18}$, and the values of $\gamma_{2}$ are calculated by solving exact recurrent equations. The line joining points is shown to guide the eyes. 


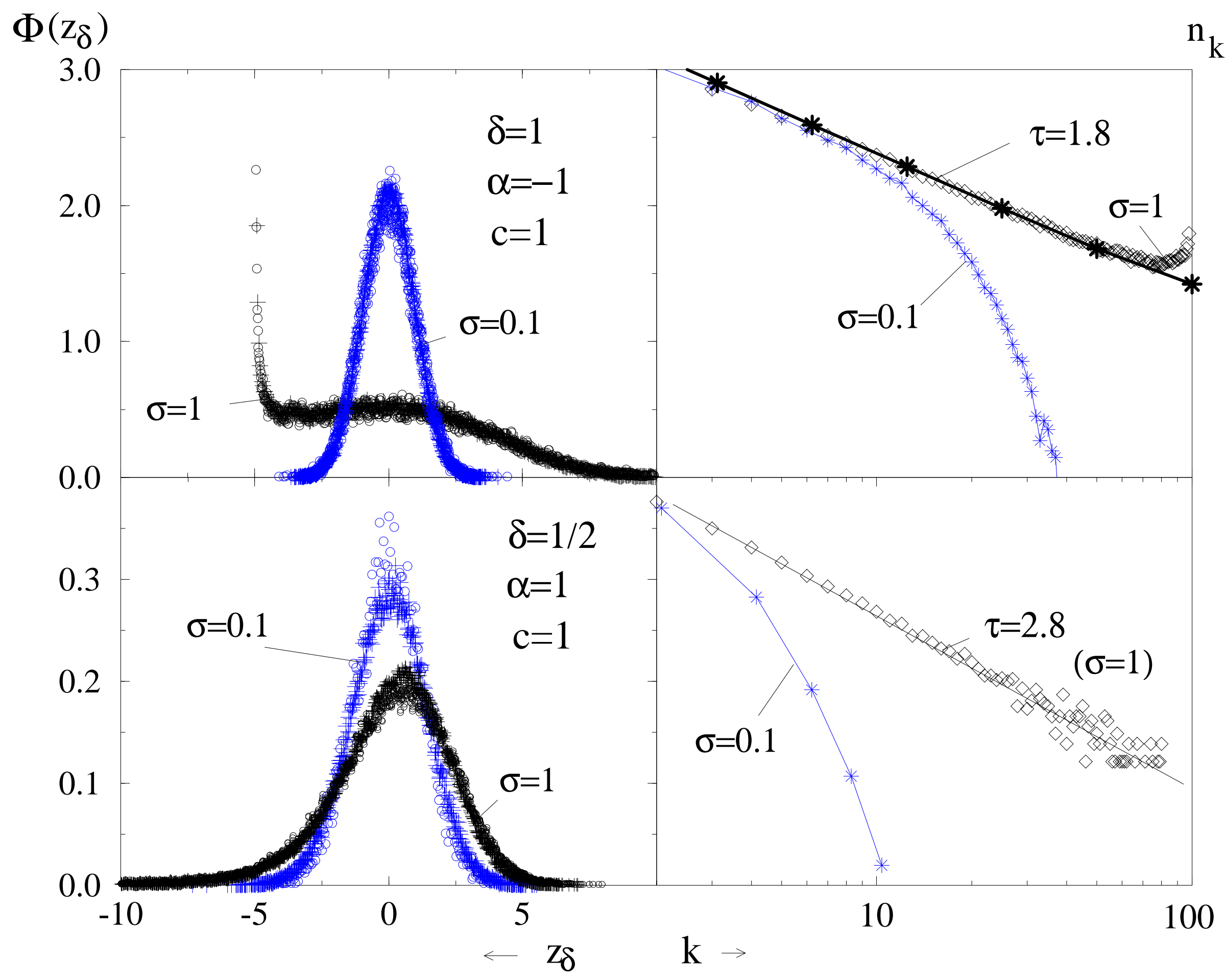




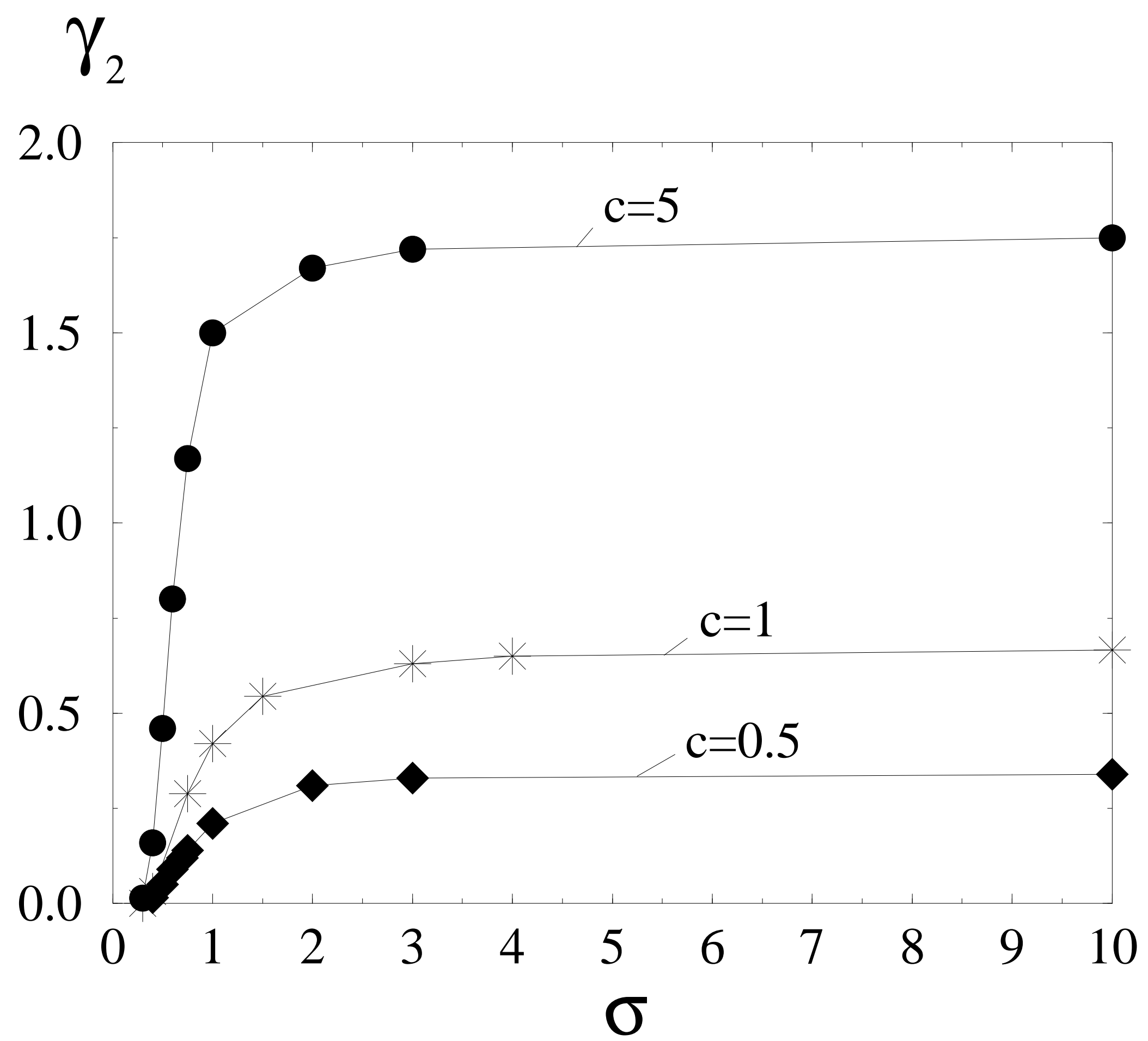

